Widefield Science and Technology for the SKA

SKADS CONFERENCE 2009

S.A. Torchinsky, A. van Ardenne, T. van den Brink-Havinga, A.J.J. van Es, A.J. Faulkner (eds.)

4-6 November 2009, Château de Limelette, Belgium

\title{
The Square Kilometre Array - an overview
}

\author{
R.T. Schilizzi ${ }^{1}$, P.E. Dewdney ${ }^{1}$, and T.J.W. Lazio ${ }^{2}$ \\ 1 SKA Program Development Office, University of Manchester, UK \\ 2 Naval Research Laboratory, Washington DC, USA
}

Abstract. A brief overview is given of the international SKA project and developments during the past year, paying particular attention to technical highlights, site characterisation, schedule and policy issues.

\section{Introduction}

The Square Kilometre Array (SKA) will be a radio interferometer with an aggregate collecting area of about 1 million square metres spread over at least $3000 \mathrm{~km}$ and operating in the frequency range $70 \mathrm{MHz}$ to $30 \mathrm{GHz}$. It is one of a small number of flagship astronomical instruments that will span the entire electromagnetic spectrum from radio to gamma rays, and beyond the electromagnetic spectrum to gravitational waves, cosmic rays and neutrinos, and whose collective aim is chart the full history of the universe from its beginnings in the Big Bang to the present day.

The SKA had its genesis in 1991 with three publications (Swarup, 1991; Noordam et al., 1991; Wilkinson, 1991) on the need for a large collecting area radio telescope to study the neutral hydrogen content in the early universe, and its subsequent evolution. Underpinning these papers was the recognition that probing the fundamental baryonic component of the Universe much beyond the local Universe would require a substantial increase in collecting area. The translation of these ideas to a concept instrument began in 1993 with the establishment of a Large Telescope Working Group by the Commission for Radio Astronomy in the International Union for Radio Science (URSI) to study the next generation radio telescope. This was followed two years later with the first allocation of funds for engineering development, in the Netherlands, for aperture array technology. The first international engineering meeting was held in Sydney in 1996, and the first agreement to collaborate on SKA technology on a global scale was also signed in 1996. The SKA has evolved over the intervening years from a simple "hydrogen array" observing at frequencies near $1.4 \mathrm{GHz}$, to a multi-facetted science facility capable of answering many of the major questions in modern astrophysics and cosmology, physics, and astrobiology.

The SKA has always been a global "grass-roots" initiative and now encompasses about 70 institutes in 20 countries. Work on the project is currently organised under an international collaboration agreement that established the SKA Science and Engineering Committee (SSEC) comprising the directors or senior representatives of 20 of those institutes and which is charged with the oversight of the science and engineering directions for the SKA. The SKA Program Development Office (SPDO) reports to the SSEC and coordinates the engineering, science, and site characterisation work carried out around the world. In addition, funding agencies and government departments of science and technology in fifteen countries discuss the SKA project on a regular basis in a group called the Agencies SKA Group (ASG).

The project has attracted substantial national and re gional funds for technical R\&D, including major design/preparatory studies in Europe (SKADS and PrepSKA), the USA (Technology Development Program) and Canada as well as the design and construction of few-percent SKA scale pathfinder telescopes in a number of countries. As a result of this work, and several internal evaluations, the original seven technical concepts for the antennas have been narrowed down over the years to four that cover the wavelength range, and enable the key science projects. These are to be found in the current system design. The project timeline has the telescope fully operational in 2023 at frequencies below $10 \mathrm{GHz}$.

A concerted effort by the science community in 2004 resulted in the publication of "Science with the SKA" (Carilli and Rawlings (eds), 2004), a 49-chapter compendium of the astrophysics and cosmology enabled by the SKA providing the motivation for constructing the telescope. It is also an outstandingly good reference book for radio astronomy in general.

The SKA will be sited in either Australia + New Zealand or in Southern Africa, with the core of the telescope in a desert region in Western Australia or the Northern Cape Province in South Africa respectively. A rigorous process led to the identification of these two sites in 2006 as being acceptable for much of the SKA's intended wavelength range. Further characterisation of the sites is underway at the present time, and current planning shows the final selection of the site taking place in 2012.

The following sections outline many of the aspects touched on in this introduction. Much of the material has been drawn from a number of key SKA publications including a comprehensive article by Dewdney et al on design considerations for the international SKA project in a special issue on Advances in Radio Telescopes in the Proceedings of the IEEE (2009), and an overview article by Schilizzi et al in Proceedings of the SPIE (2008). 


\section{Science}

From the wider science case (Carilli and Rawlings 2004), a select set of investigations - Key Science Projects - have been identified (Gaensler, 2004) in which centimetre- and metre-wavelength observations are required to make fundamental progress in outstanding questions of modern astronomy, physics, or astrobiology. The five Key Science Programs are the following.

2.3 Probing the Dark Ages: As the first stars and galaxies formed, their ionizing UV radiation produced a fundamental change in the surrounding intergalactic medium, from a nearly completely neutral state to the nearly completely ionized Universe in which we live today. The most direct probe of this era, the Epoch of Re-ionization (EoR), and of the first large-scale structure formation, will be obtained by imaging neutral hydrogen and tracking the transition of the intergalactic medium from a neutral to ionized state.

Galaxy Evolution, Cosmology and Dark Energy: Hydrogen is the fundamental baryonic component of the Universe. With a sensitivity to the 21-cm hyperfine transition of $\mathrm{HI}$ allowing detection out to redshifts $z>1$, the SKA will both follow the assembly of galaxies as well as use their HI emission as a scale tracer for cosmological probes. One of the key questions is the assembly of galaxies; the SKA will probe how galaxies convert their gas to stars over a significant fraction of cosmic time and how the environment affects galactic properties. Simultaneously, baryon acoustic oscillations (BAOs), remnants of early density fluctuations in the Universe, serve as a tracer of the early expansion of the Universe. The SKA will assemble a large enough sample of galaxies to measure BAOs as a function of redshift to constrain the equation of state of dark energy.

2.3 The Origin and Evolution of Cosmic Magnetism: Magnetic fields likely play an important role throughout astrophysics, including in particle acceleration, cosmic ray propagation, and star formation. Unlike gravity, which has been present since the earliest times in the Universe, magnetic fields may have been generated essentially ab initio in galaxies and clusters of galaxies. By measuring the Faraday rotation toward large numbers of background sources, the SKA will track the evolution of magnetic fields in galaxies and clusters of galaxies over a large fraction of cosmictime. In addition to elucidating the role of magnetic fields in galaxies and clusters of galaxies, the SKA observations will seek to address whether magnetic fields are primordial and dating from the earliest times in the Universe or generated much later by dynamo activity.

2.4 Strong Field Tests of Gravity Using Pulsars and Black Holes: With magnetic field strengths as large as $10^{14} \mathrm{G}$, rotation rates approaching $1000 \mathrm{~Hz}$, central densities exceeding $10^{14} \mathrm{~g} \mathrm{~cm}^{-3}$, and normalized gravitational strengths of order 0.4 , neutron stars represent one of the most extreme laboratories in the Universe. Their utility as fundamental laboratories has already been demonstrated through results from observations of a number of objects. The SKA will find many new milli-second pulsars and engage in high precision timing of them in order to construct a Pulsar Timing Array for the detection of nanoHertz gravitational waves, probing the spacetime environment around black holes via both ultra-relativistic bi- naries (e.g., pulsar-black hole binaries) and pulsars orbiting the central supermassive black hole in the center of the Milky Way, and probe the equation of state of nuclear matter.

2.5 The Cradle of Life: The existence of life elsewhere in the Universe has been a topic of speculation for millennia. In the latter half of the last century, these speculations began to be informed by observational data, including organic molecules in interstellar space, and proto-planetary disks and planets themselves orbiting nearby stars. With its sensitivity and resolution, the SKA will be able to observe the centimetrewavelength thermal radiation from dust in the inner regions of nearby proto-planetary disks and monitor changes as planets form, thereby probing a key regime in the planetary formation process. On larger scales in molecular clouds, the SKA will search for complex prebiotic molecules. Finally, detection of transmissions from another civilization would provide imme- $\int$ diate and direct evidence of life elsewhere in the Universe, and the SKA will provide sufficient sensitivity to enable, for the first time, searches for unintentional emissions or "leakage."

In addition, recognizing the long history of discovery at radio wavelengths (pulsars, cosmic microwave background, quasars, masers, the first extrasolar planets, etc.), the international science community also recommended that the design and development of the SKA have "Exploration of the Unknown" as a philosophy. Wherever possible, the design of $\Omega$ the telescope is being developed in a manner to allow maximum flexibility and evolution of its capabilities in new directions and to probe new parameter space (e.g., time-variable phenomena that current telescopes are not equipped to detect - radio transients). This philosophy is essential as many of the outstanding questions of the 2020-2050 era-when the SKA will be in its most productive years-are likely not even known today

\section{Design Reference Mission}

The science case has been distilled into a set of case studies called the Design Reference Mission (Lazio (ed), 2010), as a means of constraining the design specifications for the telescope. The detailed design for the SKA must be derived from the science goals but constrained by technical feasibility and cost. The DRM is a series of surveys and other observations that are sufficient to define the envelope of technical specifications; in other words, it is a subset of the full science case that sets the most exacting requirements on the technical solutions. The Design Reference Mission establishes "traceability" from the science goals to science requirements to technical requirements, and will be updated as science priorities evolve in the light of new astronomical knowledge and ongoing cost analyses.

The DRM currently contains the following case studies:

- Resolving AGN and Star Formation in Galaxies

- Pre-biotic Molecules in and around Protoplanetary Disks

- Cosmic Magnetism Deep Field, Wide Field Polarimetry

- Tracking Cosmic Star Formation: Continuum Deep Field

- Neutral Gas in Galaxies: Deep H I Field

- Epoch of Reionization: H I Imaging Tomography 
- Probing Gravity, Dark Matter, and Stellar Populations in the Galactic Center with Radio Pulsars

- Testing Theories of Gravity using Ultra-relativistic Binaries

- Tracking Galaxy Evolution over Cosmic Time via H I Absorption

- HI Baryon Acoustic Oscillations

- Pulsar Timing Array for Gravitational Wave Study

- Exploration of the Unknown: The Transient Radio Sky

- Probing AGN Environments via H I Absorption

The translation of the DRM into technical requirements is being carried out in terms of a small number of performance indicators (Dewdney et al, 2009, DRM) which guide the design and motivate the development of new technologies like the dense Aperture Arrays studied in SKADS. These parameters are: wavelength coverage, staring sensitivity, survey speed, array configuration, bandwidth, imaging and spectral dynamic range, pulsar capabilities, and time resolution.

\section{SKA System Design}

The international SKA project is currently in its "Preparatory Phase" of development. This will conclude at the end of 2012 with a costed system design for the first phase of construction and an implementation plan for the construction of the full array at frequencies below $10 \mathrm{GHz}$. Contributors to this effort are:

- SKADS - EC FP6 SKA Design Study (2005-2009), which has concentrated on the design of dense aperture arrays;

- PrepSKA - EC FP7 Preparatory Phase (2008-2011). This provides the global umbrella for the system design work (Work Package 2), site characterisation (Work Package 3, see section 5), and studies of the policy issues associated with a project of this size and cost (Work Packages 4-6, see section 6);

- US Technology Development Program (TDP, 2007-2011). The TDP is focussing on two main areas - reflector antenna development and associated single pixel feeds, and calibration and imaging with the SKA;

- SKA "precursor telescopes" on the candidate sites (MeerKAT (South Africa) and ASKAP (Australia)). MeerKAT is deploying 80 composite antennas with single pixel feeds, and ASKAP 36 panelled antennas with 3-axis rotation for phased array feeds ; and

- SKA "Pathfinder Telescopes" elsewhere (LOFAR, Apertif, ATA, MWA, LWA, EVLA, eMERLIN, eEVN). These projects are providing design and operational knowledge on low frequency sparse arrays, phased array feeds, single pixel feeds, software, and long baseline signal transport.

Major parts of the system design work coordinated by the SKA Program Development Office (SPDO) under PrepSKA have been organised into a number of Verification Programs that will deliver prototype system knowledge by 2012. These include the Dish Verification Program, the Phased Array Feed Verification Program, and the Aperture Array Verification Program. Full details of the contributions to the system design, site characterisation, and other studies by the groups around the world are contained in the revised Description of Work for PrepSKA (to be published on www . skatelescope.org).

A standard set of design reviews (conceptual design review, system specifications review, preliminary design review and critical design review) will be conducted from February 2010 onwards, and the input documentation and review panel reports will form the interim deliverables to the European Commission and to the wider community.

The current design framework for the SKA is shown schematically in Figure 1 in the form of antenna technology vs frequency vs deployment baseline. AA-lo refers to a dipole phased array or sparse aperture array, AA-hi to a dense aperture array, PAF to dishes equipped with Phased Array Feeds, and WBSPF to dishes equipped with Wide Band Single Pixel Feeds.

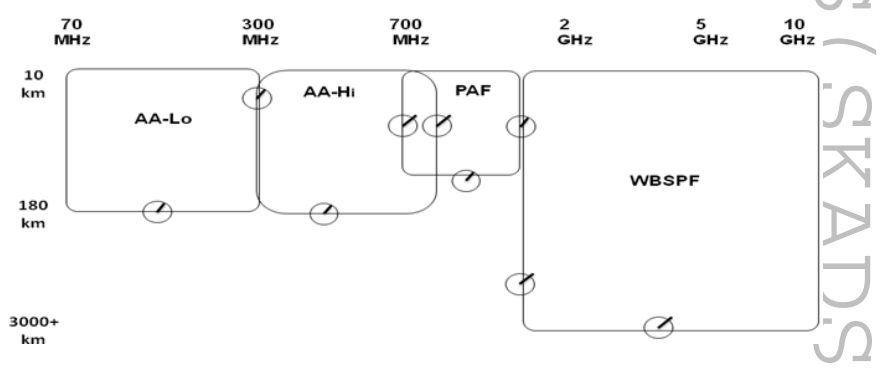

Fig. 1: The design framework for the SKA (original figure by Robert Braun and Dave Deboer).

The optimisation of technology boundaries - shown by schematic potentiometers - will take place based on science performance and cost following a trade-off analysis that includes the results of simulations of the science and technical performance.. It is unlikely that all technologies will be ready for deployment at the same time in view of their current relative levels of maturity, so that there is also a time axis underlying Figure 1 (see discussion of the SKA timeline in Section 7).

The main elements of the system design are dishes + feeds $\&$ receivers, aperture arrays, signal transport, signal processing, software engineering and algorithm development, high performance computing, data storage, and power delivery. The conceptual block diagram for the SKA is shown in Figure 2, simulations of the central cores of dense and sparse aperture arrays and dishes in Figure 3, and some pictorial highlights of the current design and prototyping efforts for antennas and feeds around the world are presented in Figures 4, 5 and 6. For an indepth discussion of these and other areas of the system design, refer to the 2009 IEEE article by Dewdney et al. 


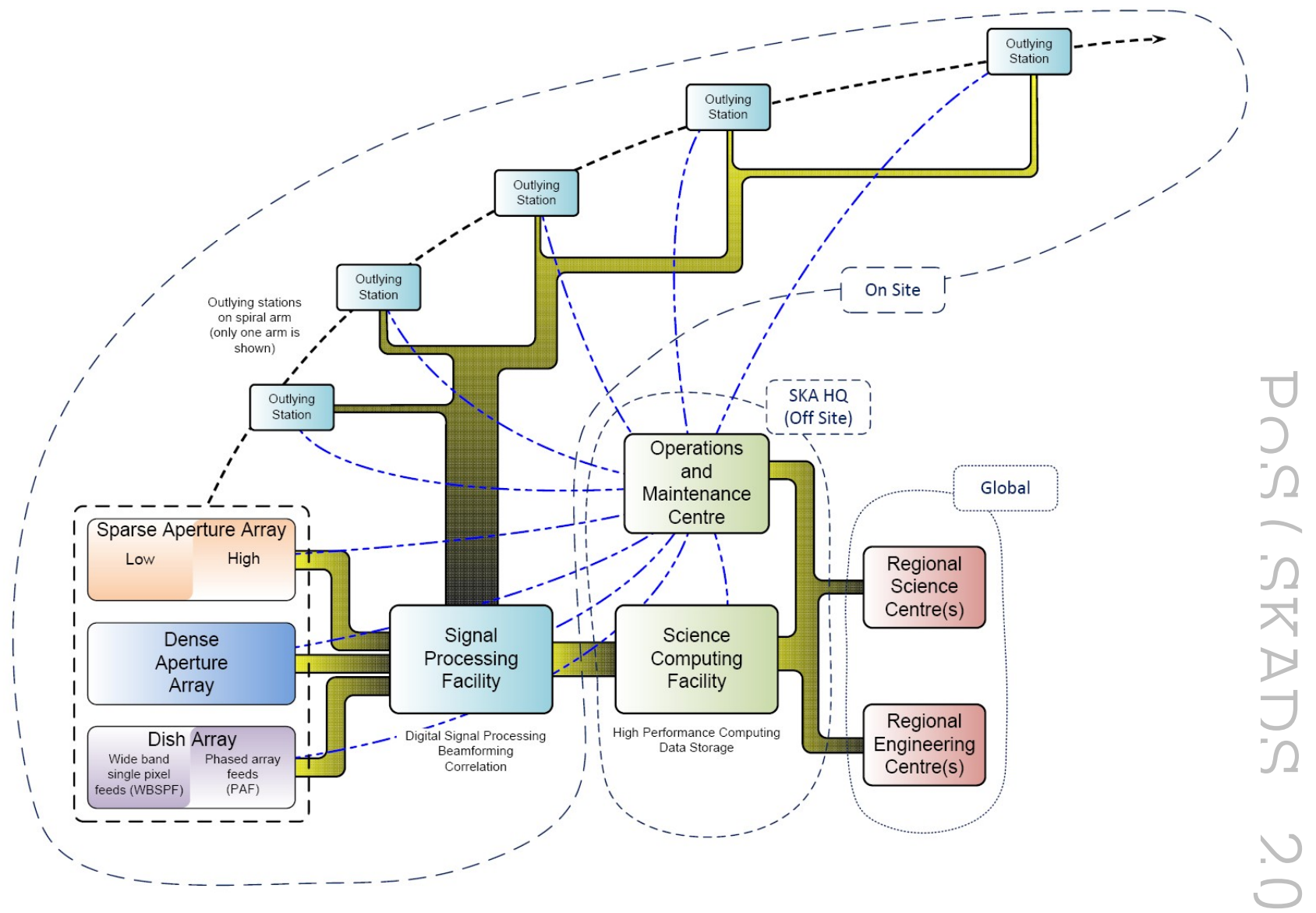

Fig. 2: A conceptual block diagram for the SKA showing the interconnection of major components, the location of major parts and the flow of data from the antennas to the left to the signal processing facility, and off-site to a computer facility. Control interconnections between the Operations and Maintenance Centre and on-site components are not shown.

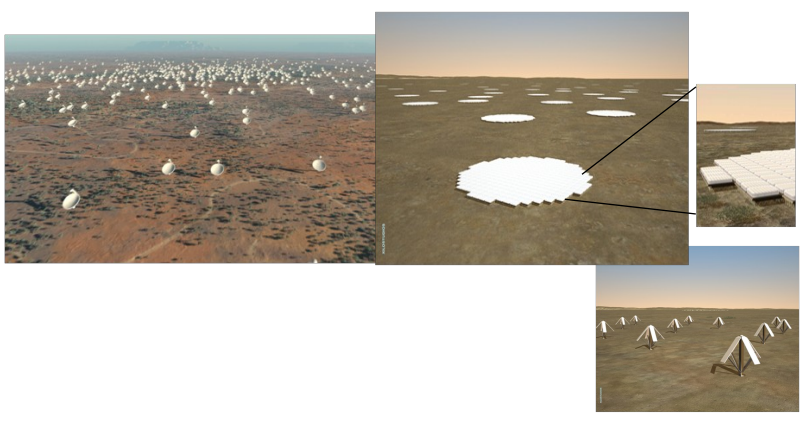

Fig. 3: Artist's impressions of the distribution of antenna elements in the three $5 \mathrm{~km}$ diameter central cores of the SKA, left dish array, middle dense aperture array, right sparse aperture array.
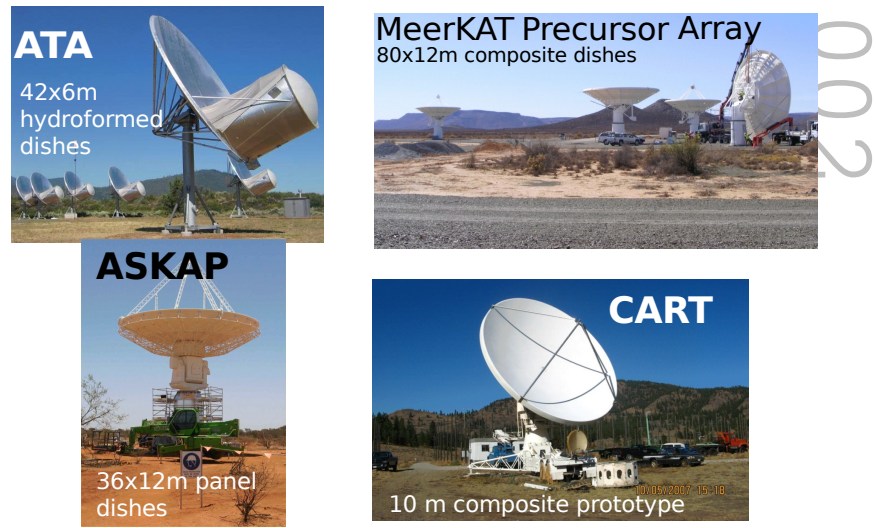

Fig. 4: Four potential dish concepts for the SKA: Allen Telescope Array 42x6m offset-fed hydro-formed dishes; MeerKAT Precursor Array $80 \times 12 \mathrm{~m}$ symmetric composite dishes; Canadian 10m symmetric composite; and Australian SKA Pathfinder 36x12m symmetric panelled dishes with 3-axis rotation. Current prototype development for the SKA system design is focussing on offset-fed $15 \mathrm{~m}$ composite dishes with accommodation for both single pixel and phased array feeds. 

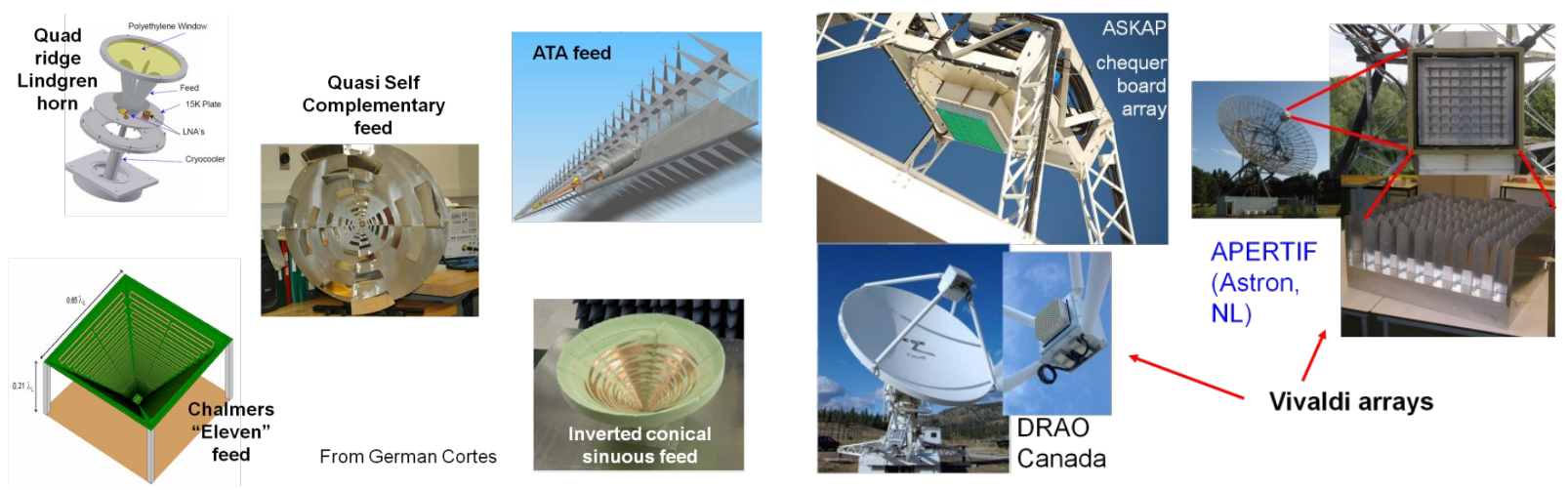

Fig. 5: (left). Examples of single-pixel, wideband feeds for reflector antennas. Clockwise from top left: Quad-ridge Lindgren horn; feed from the Allan Telescope Array; inverted conical sinuous feed; Chalmers "Eleven" feed; and, centre, Quasi Self-Complementary Antenna All of these feeds are being evaluated for use with the SKA (illustrations courtesy of G. Cortes Medellin). All are dual polarization. (right). Examples of phased array feeds for reflector antennas. Clockwise from top left: ASKAP chequer board array shown deployed on a test antenna; APERTIF Vivaldi array shown deployed on one of the Westerbork array telescopes; and the PHAD Vivaldi array shown deployed on the prototype 10m composite dish in Canada. All are dual polarisation.
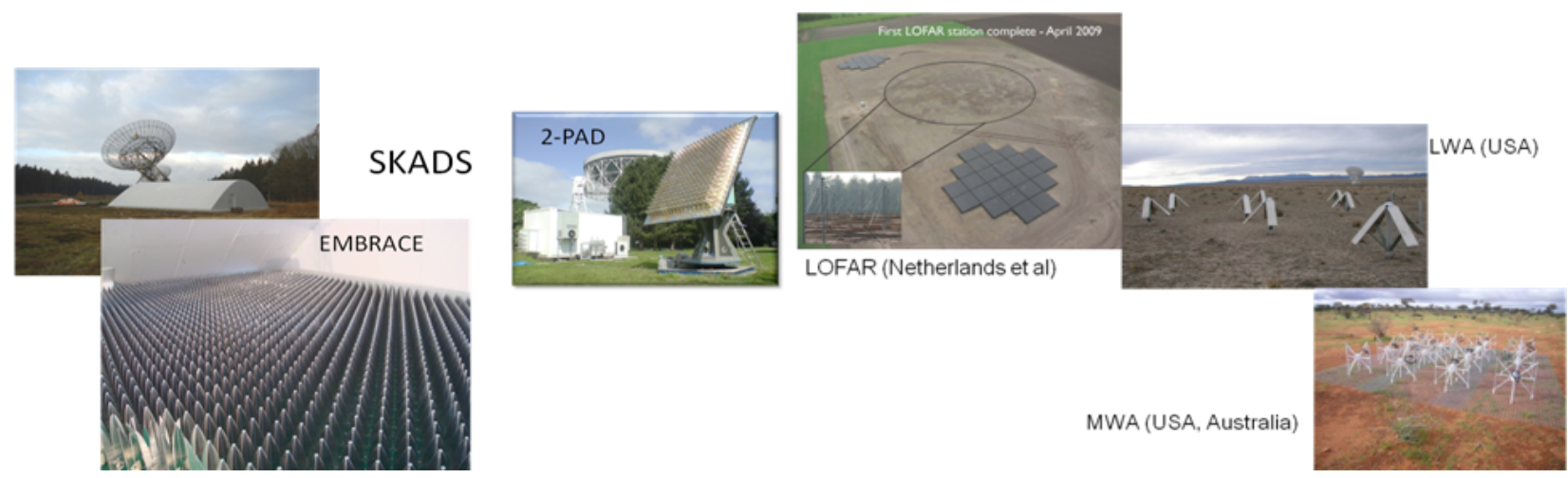

Fig. 6: (left). Dense aperture array development. Clockwise from top-left: Enclosure for EMBRACE in front of the Westerbork telescope 2-PAD array in front of the Lovell telescope at Jodrell Bank Observatory.; view of 80 square metres of the EMBRACE Vivaldi array inside the enclosure. (right). Sparse aperture array development. Top-left: aerial view of the central station for LOFAR in the Netherlands; middle - test station for the Long Wavelength Array (LWA) in the USA; bottom-right - one of the stations of the Murchison Wide-Field Array (MWA) in Australia. 


\section{Site characterisation}

Following the short-listing of sites in Western Australia and Northern Cape Province in South Africa as acceptable locations for the SKA core, a series of studies of the characteristics of these sites was instigated in PrepSKA Work Package 3, in preparation for final selection of the site in 2011-2. These studies include highly sensitive measurements of the radio quietness in the central region as well as selected remote stations, establishment of Radio Quiet Zones covering the central $200 \mathrm{~km}$ diameter region, optimization of the array configuration, the costs of infrastructure deployment and operations, the impact of physical characteristics of the sites on the design, characterisation of ionospheric turbulence, characterisation of tropospheric water vapour turbulence, and a risk analysis of the science environment with respect to long term interference potential.

Results of some of these studies are already to hand, others are in progress or are planned in the next few months. Radio Quiet Zones have been established in both countries; studies of the ionosphere have been completed; array configurations (see Figure 6) are being generated for both Australia + New Zealand and Southern Africa, taking geographic no-go zones (cities, towns, bodies of water, slopes etc) into account, as well as distances from potential sources of interference; the RFI measurement campaign is expected to start in April 2010; the tropospheric phase monitoring campaign is expected to start in late 2010; and studies of the costs of deployment of infrastructure and the long term RFI environment by external consultants will be carried out the second half of 2010.

Reports on all eight of the Work Package 3 tasks will be completed by the end of the first quarter of 2011 in order to inform the process of site selection, expected to start in mid2011.

\section{SKA governance, funding, procurement policy and industry involvement}

The Funding Agencies in the Netherlands (NWO), Italy (INAF) and the UK (STFC) are leading the development of options for governance (WP4), procurement and industry involvement (WP5), and funding (WP6) respectively, and expect to deliver final reports on these issues to the European Commission and the wider community by the end of March 2011.

In WP4, options are under consideration for the governance in the pre-construction phase after PrepSKA and the TDP replacing the SKA Science and Engineering Committee and the SPDO, as well as the long term management structure during construction and operations. Alternatives are a national legal entity, an international organisation like ITER, an MoU-based arrangement like the LHC, and a treaty-based arrangement like CERN and ESO. It is widely recognised that concluding a treaty is likely to be too time-consuming to be of use to the SKA project.

In WP5, activities include studies of global procurement options involving an analysis of WTO principles and country/ regional / international models for procurement; information gathering from Precursors/Pathfinders/ Design Studies on their industry relationships and relevant government policies; mapping and verifying potential global industry players and creating a database; investigation of current 'best practice' models for industry participation; harmonising the approach of 'regions' to the development and networking of industry capability for the SKA, and the development of an IP strategy for the SKA project.

In WP6 on funding options, thought is being given to what is needed in the different countries in terms of a business case or proposal for funding. Items already identified are the science case, industrial return, and a case for the SKA as a tool for innovation and societal benefit. A draft spending profile from 2011 to 2023 including initial phase operations ramping up from 2016 is in preparation.

\section{SKA schedule and milestones}

It appears likely that the SKA at low and mid-band frequen cies will be funded in two steps to accommodate other pressures on the public purse from scientific infrastructures. The first tranche of money will be sufficient for construction of about $10 \%$ of the array (Phase 1), with the timing of the second tranche being such as to allow a continuous rollout of the remainder (Phase 2). The current model for the division of fundsfor Phases 1 and 2 is $300 \mathrm{M} €$ and $1200 \mathrm{M} €$ respectively, in 2007 units.

\subsection{SKA schedule}

The SKA schedule can be divided into five separate stages:

A. Preparatory Phase (2007-2012) in which the telescope system design and cost for Phase 1 is completed and an implementation plan for the full SKA at these frequencies is provided. Before the end of this period, the SKA organisation will be formed once the site has been selected, and recruitment of project staff initiated.

B. Detailed design, Production Engineering \& Tooling Phase (2013-2014) in which final preparations are made for the start of Phase 1 construction in 2015. This will include additional prototyping where necessary. Site infrastructure preparation and initial rollout will occur. System design of the less mature technologies not included in Phase 1, will continue throughout this period.

C. Phase 1 Construction, Verification, Commissioning, Acceptance, Integration \& First Science (2015-2018) in which Phase 1 is rolled out and integrated and first science is carried out as part of the commissioning process, starting in 2016 with a sub-array of Phase 1. Final selection of Phase 2 technology will take place in 2015 followed in 2016 and 2017 by detailed design and construction preparation for those design elements not in Phase 1.

D. Phase 2 Construction, Verification, Commissioning, Acceptance, Integration \& First Science (2017-2022) in which the construction of the SKA is completed and first science is carried out as part of the commissioning period.

E. SKA operations begin $(2023 \longrightarrow)$ 

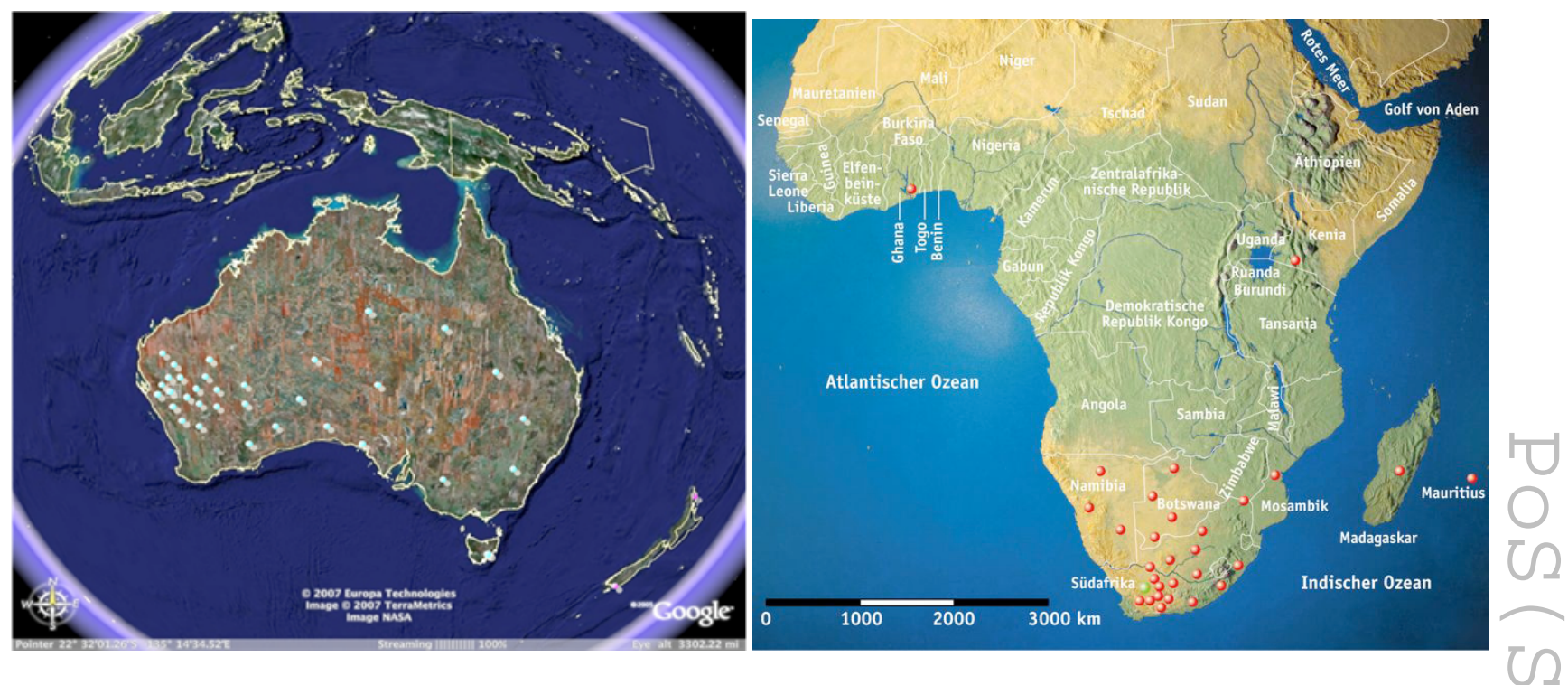

Fig. 7: Representative configurations for the SKA in Australia + New Zealand and for Southern Africa. Detailed configurations are currently being developed. In the central region of the SKA (not shown in detail) three separate cores are required, for the dish array, the dense aperture array and the sparse aperture array.

\subsection{Planned intermediate milestones}

2011 funding for 2012-2014 (pre-construction) approved 2012 site selection made, and recruitment for the detailed design and construction phase is initiated

2013 initial (Phase 1) construction funding approved

2016 construction funding approved for the full SKA at mid and low frequencies

2019 science operations with Phase 1 commences

\subsection{High frequency component of the SKA}

Current planning foresees 2013 to 2022 being used for high frequency technology development and prototyping, followed, in 2023 by the start of construction of the high frequency component of the SKA.

\section{Costs}

As noted in the previous section, the target construction cost is 1.5 billion $€(2007)$ for Phases $1+2$. This includes civil works, antennas \& RF systems, signal transmission, signal processing, software development \& computing hardware, design, integration, testing, and management, and contingency

The expected operating costs are 150 million $€ /$ year (2007) including salaries (400-500 staff), power, materials \& services including dark fibre lease, and renewal of instrumentation and computing. The costs of regional science centres are additional.

\section{References}

Carilli C. and Rawlings S. (eds), "Science with the SKA", 2004 New Astronomy Reviews, Vol. 48, Nos 11-12, pp 979-1606

Dewdney, P.E., Hall, P.J., Schilizzi, R.T., Lazio, T.J.W., 2009, Proceedings of the IEEE, vol 97, No 8, 1482-1496

Lazio, T.J.W. (ed.) and the Science Working Group, 2010, to be published on www. skatelescope.org

Noordam, J.E., Braun, R., de Bruyn, A.G., 1991, Netherlands Foundation for Radio Astronomy Note nr 585 (available on www. skatelescope.org)

Schilizzi, R.T., Dewdney, P.E., Lazio, T.J.W., 2008, Proceedings of the SPIE, vol 7012, 7012111-13

Gaensler, B.M. 2004, "Key Science Projects for the SKA" SKA Memorandum 44 (www. skatelescope. org)

Swarup G., 1991, Current Science, 60 (2), 106-108

Wilkinson, P.N., 1991, Proceedings of IAU Colloquium 131, ASP Conference Series, Vol. 19, T.J. Cornwell and R.A. Perley (eds.), 428-432. 